Proceedings

\title{
Advanced Energy Harvesting from Heating for a Water Consumption Meter ${ }^{+}$
}

\author{
Zdenek Machacek ${ }^{1}$, Vojtech Sotola ${ }^{1}$, Radek Petras ${ }^{1}$, Miroslav Schneider ${ }^{1}$, Zdenek Masny ${ }^{2}$, \\ Adam Idzkowski ${ }^{3}$ and Wojciech Walendziuk ${ }^{3, *}$ \\ 1 Department of Cybernetic and Biomedical Engineering, VSB Technical University of Ostrava, \\ 17.listopadu 15, 70800 Ostrava, Czech Republic; zdenek.machacek@vsb.cz (Z.M.); \\ vojtech.sotola@vsb.cz (V.S.); radek.petras@outlook.cz (R.P.); miroslav.schneider@vsb.cz (M.S.) \\ 2 Company CODEA, Premyslovcu 30, 70900 Ostrava, Czech Republic; codea@codea.cz \\ 3 Faculty of Electrical Engineering, Bialystok University of Technology, Wiejska 45D, 15-351 Bialystok, \\ Poland; a.idzkowski@pb.edu.pl \\ * Correspondence: w.walendziuk@pb.edu.pl; Tel.: +48-85-7469397 \\ + Presented at the 9th Innovations-Sustainability-Modernity-Openness Conference (ISMO'20), Bialystok, \\ Poland, 20-21 May 2020.
}

Published: 4 August 2020

\begin{abstract}
This study focused on the design of the power supply and the overall control structure of the electronic system, which is charged only with energy from the environment. It describes the basic finding of all the necessary information for the design and implementation with the measurement and evaluation of characteristic parameters. The paper describes the individual possibilities of obtaining energy from the surroundings with the selected energy from the heat of the pipeline. There are also various arrangements of possible elements mentioned in order to ensure the optimal solution of the whole system. Furthermore, a basic evaluation of the comparison of a possible workable solution is made.
\end{abstract}

Keywords: DC-DC power converters; energy consumption; energy harvesting; energy measurement; supercapacitors; water flowmeter

\section{Introduction}

The paper describes a proposal for the most optimal power system solution to measure the flow of media (e.g., water) without the usage of batteries. The energy needed to power the system should be "recovered" from the environment or from the medium being measured [1].

First, a general analysis of the possibilities for energy-harvesting resources was carried out with regard to the possibilities of heat utilization in the cases of measuring heat supplies. Another implemented alternative way of obtaining electricity is to convert solar energy. This transformation occurs in solar cells, which can be found in pocket calculators. Another option is the use of temperature difference. Specialized thermocouples are able to convert the temperature difference into electricity. The direct conversion of mechanical energy into electrical elements containing special substances capable of a piezoelectric effect or induction elements can be another method $[2,3]$.

\section{Energy-Harvesting System}

This section of the paper contains the basic division of topologies and describes the individual topologies, showing the differences between them. Subsequently, the elements used in the topologies are theoretically described here. Each topology includes a selected power source that delivers the electrical power with certain parameters. These parameters mostly do not match the parameters that 
require the load (appliance), so the electrical energy from the source must be converted into the electrical energy demanded by the appliance. This conversion occurs in special drives. If the source of the supply is DC power, in the majority of cases a DC/DC converter is usually used. If the AC power supply is alternating, it supplies AC power, and the AC/DC converter is used. However, AC sources are less frequent than DCs, so the use of AC/DC converters is not very common [4].

Within the design, the power system was divided into three possible basic configurations. Each layout has its advantages and disadvantages, so it is necessary to analyze the most optimal solution well.

The easiest design is shown in Figure 1a. It uses only one DC/DC converter, so a smaller number of components leads to a higher reliability (less fault) and lowest price. The second option is to add the possibility of the accumulation of electrical energy in Figure 1b, in particular in a supercapacitor. Thus, it is possible to cover a longer outage. The disadvantage is the usage of two DC/DC converters, resulting in lower efficiency of the total wiring (at the efficiency of DC/DC converters of $80 \%$, total efficiency is $64 \%$ ). The third option is shown in Figure 1c. Compared to the second option, it also includes a primary cell (battery) that is needed in systems that need to know the time and date, even in the case of a power failure. Unfortunately, this is the biggest drawback of this concept because the primary cell has a limited lifetime and must change at regular intervals.

The requirements of a non-battery solution based on a thermoelectric generator (TEG cell) was evaluated. This second variant (Figure 1b) seems to be the most optimal solution in which energy is accumulated without the primary cell with the use of the supercapacitor.

a)

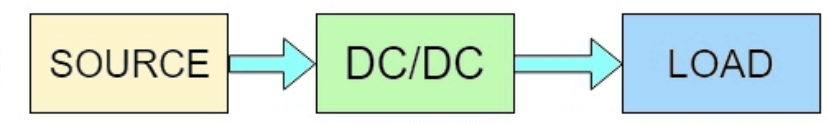

b)

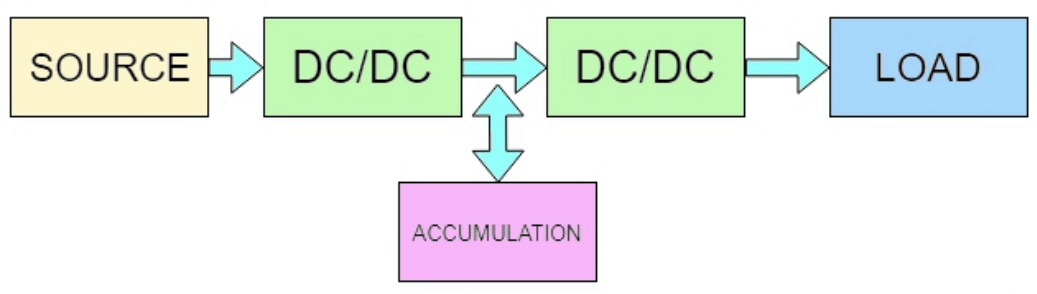

c)

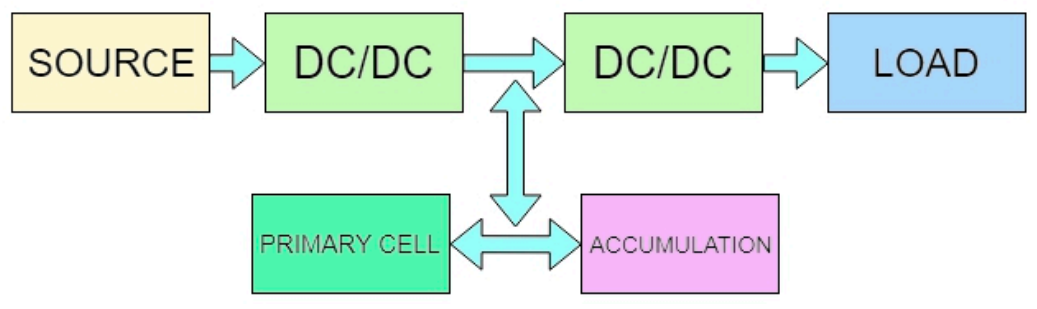

Figure 1. Supply system topology (a - direct system, $\mathbf{b}-$ accumulator system, $\mathbf{c}-$ battery system).

\section{Analysis of the Developed System Power Supply}

By measuring the tested TEG cell, it has been found that such a power supply could be used just in applications meeting the following criteria:

- the power supply must represent a load equivalent to the test load shown in the chart;

- $\quad$ the TEG cell must be applied at a sufficient temperature difference $\Delta \mathrm{T}[\mathrm{K}]$.

When the criteria are met, a TEG cell with a suitable DC/DC converter can be used as a permanent source of power supply to the application sensor (Figure 2). An appropriately selected application (e.g., MCU power supply) would allow the TEG cell to feed from the temperature difference $\Delta \mathrm{T}=3 \mathrm{~K}$. In the research, such a temperature difference is sufficient to supply the circuit in the case when the high impedance load is used. At a lower impedance load, it would be necessary to provide a higher temperature difference on the TEG cell according to the data in the graph (Figure 
3). It is worth stressing that the whole electronics with a microcontroller must be supplied by power of at least $518 \mu \mathrm{A}$ for $3.3 \mathrm{~V}$ and $350 \mu \mathrm{A}$ for $2.2 \mathrm{~V}$. The water consumption process was realized then with a frequency of eight measurements per second. These power consumption parameters were achieved by optimizing the microprocessor state from the active mode to the low power mode.

It is apparent from the graphs (Figure 3) that even a developed functional consumption meter can be permanently powered from the temperature difference $7 \mathrm{~K}$.

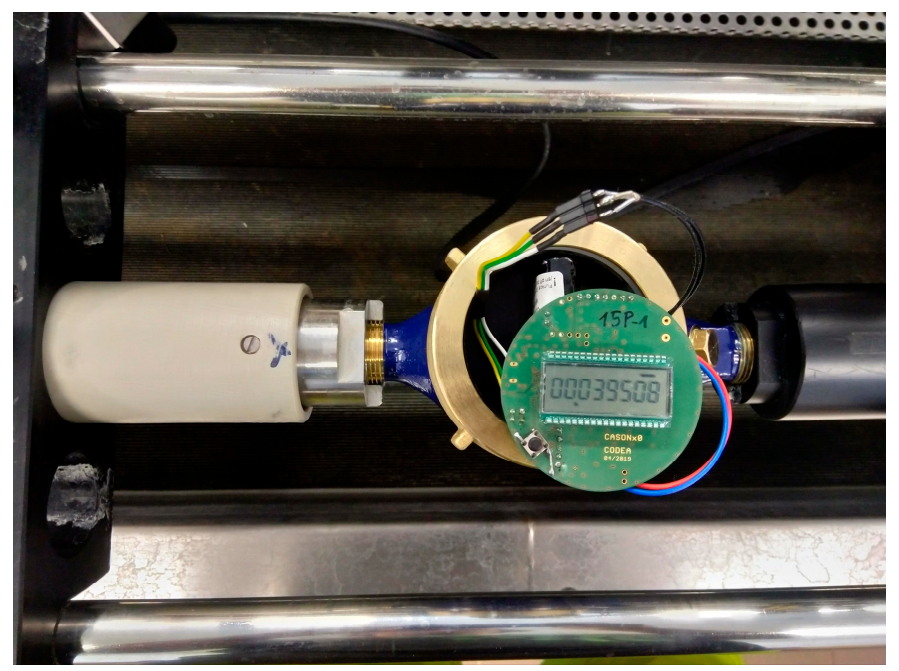

Figure 2. General view of the measured system, equipped with a flowmeter, used in the research.

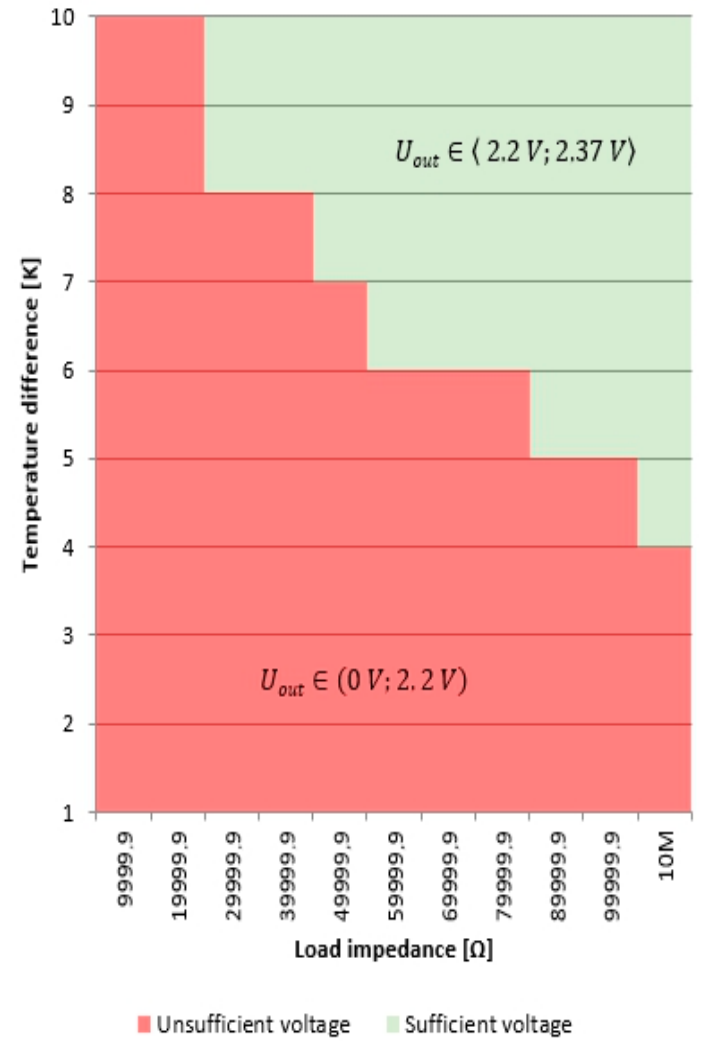

(a)

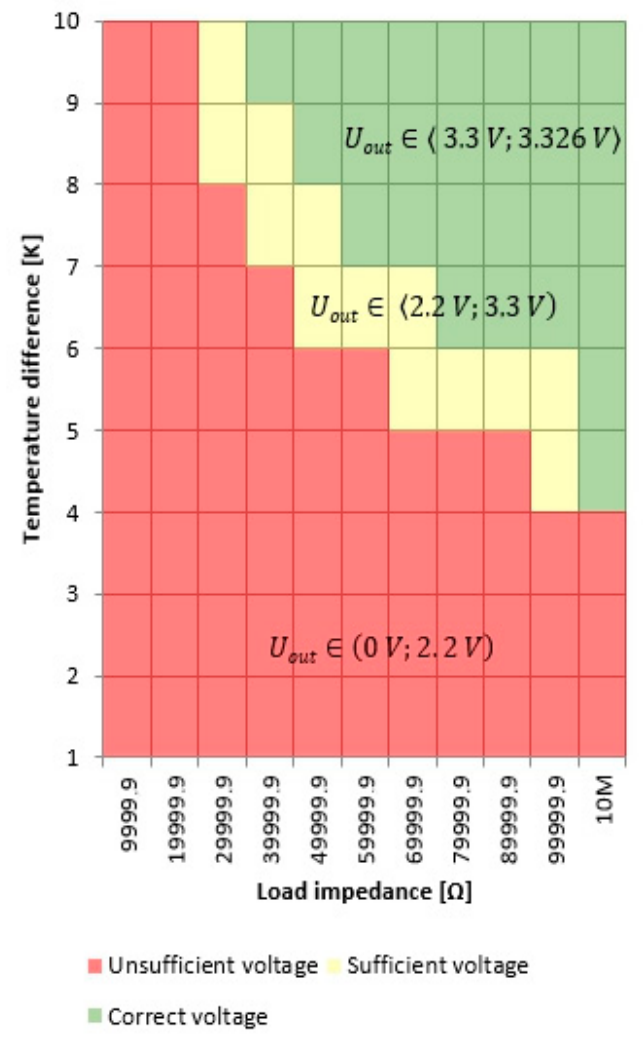

(b)

Figure 3. Graph of the temperature-load characteristics, dependent on the load impedance for a voltage rating: $2.2 \mathrm{~V}(\mathbf{a})$ and $3.3 \mathrm{~V}(\mathbf{b})$. 


\section{Conclusions}

The measurement and analysis of the generated power on thermocouples (TEG) is presented in Figure 3. The measurement is important to determine the sufficient power from the temperature difference to supply the device equipped with the microcontroller to measure the flow rate of the medium. The generated power is represented in the graphs by the value of the generated voltage and the value of the impedance, from which the generated current of the electric circuit can be calculated. The measurement was conducted for two voltage level limit values, which allows power to supply the electronics and the microcontroller. The designed and realized converter is always optimized for the given voltage level. In conclusion, the conducted research, presented in a form of graphs, shows the areas where the green area reaches the required voltage $(2.2 \mathrm{~V}$ or $3.3 \mathrm{~V})$, the yellow area shows the minimum functional voltage $2.2 \mathrm{~V}$, and the red area does not reach the minimum required voltage of $2.2 \mathrm{~V}$.

Author Contributions: Concept of the research: Z.M. (Zdenek Machacek), V.S. and Z.M. (Zdenek Masny); methodology: V.S. and M.S.; software: R.P.; validation: V.S., M.S. and Z.M. (Zdenek Machacek); visualization: M.S., A.I., R.P. and W.W.; investigation: Z.M. (Zdenek Machacek), V.S., R.P.; resources: Z.M. (Zdenek Machacek); data curation: V.S.; writing - original draft preparation: A.I., Z.M. (Zdenek Machacek), W.W. and M.S.; writing review and editing: A.I. and W.W.; Supervision: Z.M. (Zdenek Machacek); project administration: Z.M. (Zdenek Masny); funding acquisition: Z.M. (Zdenek Machacek); W.W. and A.I. writing-review and editing; Z.M. (Zdenek Machacek) and V.S. conceived and designed the experiments; V.S. performed the experiments; M.S., W.W., A.I. and R.P. analyzed the data; V.S., M.S. and Z.M. (Zdenek Masny) contributed reagents/materials/analysis tools; Z.M. (Zdenek Machacek), V.S., W.W., A.I. wrote the paper. All authors have read and agreed to the published version of the manuscript.

Acknowledgments: This research was funded by the project CZ.01.1.02/0.0/0.0/15_019/0004955 "Energy Harvesting from environment free of Battery Supply to Measuring energy consumption in the SMART Grid Network" of Operational Program of Business and Innovation for Competitiveness and project SP2020/42, "Development of algorithms and systems for control, measurement and safety applications VI" of Student Grant System, VSB-TU Ostrava, the research has been supported by project WZ/WE-IA/2/2020 of the Bialystok University of Technology and financed from a subsidy provided by the Ministry of Science and Higher Education.

Conflicts of Interest: The authors declare no conflict of interest.

\section{References}

1. Mihajlovic, Z.; Milosavljevic, V.; Joza, A.; Rajs, V.; Damnjanovic, M.; Zivanov, M. Surface and Underground Water Level Monitoring Using Wireless Sensor Node with Energy Harvesting Support. Elektron. Elektrotech. 2016, 22, 62-68.

2. Kelnar, M.; Martinek, R.; Machacek, Z.; Vanus, J.; Bilik, P.; Zidek, J. Realization of prototype of a low-cost bidirectional communication system through fibreless optics. Wirel. Pers. Commun. 2016, 22, 62-68.

3. Ghamari, M.; Soltanpur, C.; Cabrera, S.; Romero, R.; Martinek, R.; Nazeran, H. Design and prototyping of a wristband-type wireless photoplethysmographic device for heart rate variability signal analysis. In Proceedings of the 2016 38th Annual International Conference of the IEEE Engineering in Medicine and Biology Society, Orlando, FL, USA, 16-20 August 2016; NIH Public Access: Bethesda, MD, USA, 2016; Volume 2016; p. 4967.

4. Martinek, R.; Brablik, J.; Soustek, L.; Kolarik, J.; Fajkus, M.; Nedoma, J.; Kahankova, R. A comparison of probes based on Bragg grating sensor and microphones for heart sounds measurement. In Optical Materials and Biomaterials in Security and Defence Systems Technology XV; International Society for Optics and Photonics: Washington, DC, USA, 2018; Volume 10801, p. 108010G. 